\title{
IKK $\alpha$ contributes to ischemia-induced autophagy after acute cerebral ischemic injury
}

\author{
Wen Dong, Qingfang Chen, Shunying Zhao, Shaohong Wen, Wentao Chen, Weizhen Ye, Ting Gong, \\ Mingyu Jiang, Xiangrong Liu
}

China National Clinical Research Center for Neurological Diseases, Beijing Tiantan Hospital, Capital Medical University, Beijing, China

Contributions: (I) Conception and design: W Dong, X Liu; (II) Administrative support: X Liu; (III) Provision of study materials or patients: Q Chen, S Zhao; (IV) Collection and assembly of data: S Wen, W Chen; (V) Data analysis and interpretation: W Dong, X Liu; (VI) Manuscript writing: All authors; (VII) Final approval of manuscript: All authors.

Correspondence to: Xiangrong Liu. China National Clinical Research Center for Neurological Diseases, Beijing Tiantan Hospital, Capital Medical University, No. 119 South Fourth Ring West Road, Fengtai District, Beijing 100070, China. Email: 1xrpumc@163.com.

Background: The function of IкB kinase $\alpha(\mathrm{IKK} \alpha)$ in the brain is largely unknown. This study examined the effects of IKK $\alpha$ on autophagy after cerebral ischemia.

Methods: Permanent distal middle cerebral artery occlusion (dMCAO) was conducted in C57/BL6 mice. Oxygen-glucose deprivation/reperfusion (OGD/R) was performed to mimic ischemia injury in neuro-2A (N2A) cells in vitro. Autophagy activation was assessed by detecting the ratio of microtubule-associated protein 1 light chain $3 \beta$ (LC3B)-II/LC3B-I and Cyto-ID autophagic fluorescence. The infarct volume was verified by 2,3,5-triphenyltetrazolium chloride (TTC) staining and magnetic resonance imaging (MRI). Neurological functions were evaluated using the modified Garcia test. Cell death after dMCAO was confirmed with terminal deoxynucleotidyl transferase (TdT)-mediated dUTP nick end labeling (TUNEL) assay. To determine the role of IKK $\alpha$, small interfering RNA (siRNA) was transfected into N2A cells or injected intracerebroventricularly.

Results: IKK $\alpha$ and LC3B II/I expression levels were increased both in OGD/R treated N2A cells and dMCAO mice. Under the same conditions, IKK $\beta$ expression was not altered. IKK $\alpha$ siRNA significantly decreased the infarct volume and the apparent diffusion coefficient (ADC) related to brain edema, and promoted the neurological outcomes after dMCAO. Furthermore, inhibition of IKK $\alpha$ attenuated ischemiainduced the conversion of LC3B I to LC3B II both in vitro and in vivo. In addition, IKK $\alpha$ siRNA alleviated the formation of autophagic vacuoles and LC3 positive puncta after cerebral ischemia.

Conclusions: These findings indicate that IKK $\alpha$, but not IKK $\beta$, plays a critical role in ischemia-induced autophagy. Inhibition of IKK $\alpha$ protects the brain from ischemia injury and this may have potential benefits in stroke therapy.

Keywords: IкB kinase $\alpha$ (IKK $\alpha$ ); cerebral ischemia; autophagy; neuroprotection

Submitted Jan 16, 2022. Accepted for publication Feb 18, 2022.

doi: $10.21037 / \mathrm{atm}-22-517$

View this article at: https://dx.doi.org/10.21037/atm-22-517

\section{Introduction}

Stroke is a leading cause of mortality and disability worldwide, placing significant burden on the social healthcare system (1). Tissue-type plasminogen activator (tPA) is currently the only medical treatment for acute ischemic strokes approved by the American Food and Drug Administration. However, less than $5 \%$ of ischemic patients benefit from tPA therapy due to its narrow therapeutic time window and potential severe adverse events including brain edema and the risk of hemorrhagic transformation $(2,3)$. Therefore, novel therapeutic targets for the treatment of 
ischemic injury and its related mechanisms are urgently being investigated.

The IкB kinase (IKK) complex contains two catalytic subunits, IKK $\alpha$ and IKK $\beta$, that play important roles in triggering nuclear factor $(\mathrm{NF})-\kappa \mathrm{B}$ activation under physiological and pathological conditions. While IKK $\alpha$ and IKK $\beta$ share partial structural similarities, they activate NF$\kappa \mathrm{B}$ through different mechanisms (4). Notably, both IKK $\alpha$ and IKK $\beta$ exert crucial NF- $\kappa \mathrm{B}$-independent functions through other substrates, such as c-Fos, p85a, 14-3$3 \delta$, silencing mediator for retinoid or thyroid-hormone receptors (SMRT), mammalian target of rapamycin (mTOR), steroid receptor coactivator 3 (SRC3), and maspin (4-7). Together, these studies suggest that both IKK $\alpha$ and IKK $\beta$ may act as multifunctional proteins that trigger a series of NF- $\mathrm{KB}$-independent signaling events beyond their well-known functions in the NF- $\kappa \mathrm{B}$ pathway. A previous study has shown that activation of IKK $\beta$ enhances the ischemia infarct volume at 48 hours after the onset of middle cerebral artery occlusion (MCAO) (8). In transient focal cerebral ischemia, IKK $\alpha$ is involved in oxidative stress injury and microglial polarization after stroke $(9,10)$. However, the role of IKK $\alpha$ in permanent cerebral ischemia remains unclear.

Autophagy is a highly conserved and complex catabolic process that maintains intracellular homeostasis through degradation of cytoplasmic components (macromolecules or damaged organelles) $(11,12)$. Autophagy is generally induced by stress, such as nutrient starvation, oxygen and energy deprivation $(13,14)$. Various indicators can be used to detect autophagic activity, such as the conversion of microtubule associated protein 1 light chain 3 (LC3) I to LC3 II, Beclin 1 upregulation, and SQSTM1/p62 degradation. Among these proteins, LC3 II turnover is the most widely used maker for autophagosomes $(14,15)$. The role of autophagy after cerebral ischemia is still controversial (16). It is reported that autophagy activation alleviated global ischemia induced neuronal death (17). In cultured cortical neurons, autophagy inducer rapamycin protected against neuronal damage after oxygen-glucose deprivation (OGD) treatment (18). However, inhibition of overactivated autophagy by Sodium hydrosulfide (NaHS) was reported to attenuate cerebral ischemia/reperfusion injury (19). In our study, we proposed that autophagy activation contributed to permanent cerebral ischemia injury.

In transient cerebral ischemic-reperfusion, autophagy dependent IKK $\alpha$ degradation is involved in microglial polarization (10). However, the relationship between IKK $\alpha$ and autophagy is unclear after permanent cerebral ischemia. This current study investigated the potential role of IKK $\alpha$ in mediating cerebral ischemia injury via regulating autophagy in a cellular oxygen-glucose deprivation/ reperfusion (OGD/R) model and a permanent cerebral ischemic mice model. We present the following article in accordance with the ARRIVE reporting checklist (available at https://atm.amegroups.com/article/view/10.21037/atm$22-517 / \mathrm{rc})$.

\section{Methods}

\section{Animal models and experimental groups}

All experimental procedures were performed under a project license (No. 201902039) by the Animal Welfare Ethics Committee of Beijing Neurosurgical Institute and were performed in accordance with the guidelines of the National Institutes of Health on the care and use of laboratory animals. Male C57/BL6 mice were housed individually in polycarbonate cages in a regular 12-hour light-dark cycle at $22 \pm 2{ }^{\circ} \mathrm{C}$.

Distal MCAO (dMCAO) was performed by electrocoagulation of the distal middle cerebral artery (MCA) as previously reported $(20,21)$. To confirm the ischemia in mice, regional cerebral blood flow (rCBF) was detected with a laser Doppler flowmetry (RWD Life Science, Shenzhen, China) before and after $\mathrm{dMCAO}$. The body temperature of the mice was maintained at normothermia during surgery with a heating pad (RWD Life Science).

A total of 60 mice (age of 8 to 10 weeks) weighed $24 \pm 0.5 \mathrm{~g}$ were used in this study. The mice were randomly assigned into four groups, namely, sham $(\mathrm{n}=10), \mathrm{dMCAO}$ $(\mathrm{n}=10), \mathrm{dMCAO}+$ control small interfering RNA (control siRNA; $\mathrm{n}=21)$, dMCAO + IKK $\alpha$ siRNA ( $=19)$. Surgery, siRNA preparation and administration, outcome assessment, and data analyses were performed by researchers who were blinded to the experimental conditions of the mice. No mice died due to surgery or stroke complications. A protocol was prepared before the study without registration.

\section{Cell culture and transfection}

The mouse neuroblastoma cells neuro-2A (N2A) were grown in Dulbecco's modified Eagle's medium (DMEM) supplemented with $10 \%$ fetal bovine serum. Cells were transfected with IKK $\alpha$ siRNA (CCGGGAACUUGAUCUCAAA) or control siRNA 
(UUCUCCGAACGUGUCACGU, GenePharma, Suzhou, China) using Lipofectamine ${ }^{\mathrm{TM}}$ RNAi MAX (Invitrogen, Waltham, MA, USA) according to the manufacturer's instructions.

\section{$O G D / R$}

To mimic ischemia in vitro, N2A cells were exposed to OGD for 1 hour, followed by reoxygenation for 24 hours. Briefly, the culture medium was replaced with glucose-free DMEM (Gibco, Grand Island, NY, USA) and cells were placed in a hypoxic chamber (Billups Rothenberg, San Diego, CA, USA) containing $5 \% \mathrm{CO}_{2}, 0.02 \% \mathrm{O}_{2}$ and $94.98 \% \mathrm{~N}_{2}$ according to our previous study (22). After 1 hour of OGD, cells were maintained in original culture medium for 24 hours. Cells in the control group were maintained in normal growth medium under normoxic culture conditions.

\section{Western blot assay}

Total proteins were extracted from N2A cells with lysis buffer or from mice cortex as previously described (23). All protein blots were probed with primary antibodies against IKK $\alpha$, IKK $\beta$, microtubule associated protein 1 light chain $3 \beta$ (LC3B), or actin $(1: 1,000$, Cell Signaling Technology, Beverly, MA, USA). Protein expression was quantified and normalized to actin, the loading control.

\section{Cyto-ID autophagy detection}

To detect specific autophagic fluorescence signals in N2A cells, the Cyto-ID Autophagy Detection Kit was used according to the manufacturer's instructions (Enzo Life Science, Farmingdale, New York, USA). Briefly, after treatment, N2A cells were carefully washed twice with assay buffer and incubated with Cyto-ID green detection reagent and 4',6-diamidino-2-phenylindole (DAPI) nuclear stain for 30 minutes at $37^{\circ} \mathrm{C}$. Cells were then washed twice with assay buffer. The stained cells were observed by confocal microscopy (Zeiss, Oberkochen, Germany) using a FITC filter (autophagic signal) and a DAPI filter.

\section{Intracerebroventricular (i.c.v) injection}

IKK $\alpha$ siRNA or control (Con) siRNA $(5.6 \mu \mathrm{L})$ mixed with the transfection reagent $(1.4 \mu \mathrm{L}$, Invitrogen) was delivered into the right ipsilateral ventricle $(1.0 \mathrm{~mm}$ lateral and
$0.5 \mathrm{~mm}$ posterior to bregma, $2.5 \mathrm{~mm}$ dorsoventral below the skull) according to the stereotaxic map in the mice 1 hour before $\mathrm{dMCAO}$ onset. The injection was administrated at a rate of $0.5 \mu \mathrm{L} / \mathrm{min}$ and the needle was retained for another 15 minutes after injection. The bone wound was sealed with bone wax.

\section{Immunofluorescence staining}

Mice brain sections $(20 \mu \mathrm{m})$ were stained with rabbit antiLC3 (autophagy marker) and DAPI (a nuclear specific marker) and observed with a confocal microscope (Zeiss).

\section{2,3,5-triphenyltetrazolium chloride (TTC) staining}

Mice were deeply anesthetized using isoflurane at 24 hours after dMCAO. The brains were removed quickly and cut into seven $1-\mathrm{mm}$ thick slices. The infarct volume was measured by TTC staining as described previously (20).

\section{Magnetic resonance imaging (MRI)}

MRI was conducted using a 7.0 $\mathrm{T}$ BioClinScan animal system (Bruker, Germany) to assess the extent of brain infarction and water diffusion. The $\mathrm{T} 2$ weighted (T2W) sequence consisted of the following parameters: time to repetition/echo time (TR/TE) $=3,080 \mathrm{~ms} / 41 \mathrm{~ms}$, field of view $(F O V)=24 \mathrm{~mm} \times 30 \mathrm{~mm}$, and slice thickness $=0.5 \mathrm{~mm}$. The diffusion weighted sequence was performed using the following parameters: TR/TE $=4,500 \mathrm{~ms} / 46 \mathrm{~ms}$, $\mathrm{FOV}=25 \mathrm{~mm} \times 30 \mathrm{~mm}, \mathrm{~b}$ value $=600 \mathrm{~s} / \mathrm{mm}^{2}$, number of slices $=28$. Mice were anesthetized using isoflurane $(1.5 \%)$ during the MRI experiments at 24 hours after dMCAO. The infarct areas after $\mathrm{dMCAO}$ showed high intensity signals on $\mathrm{T} 2 \mathrm{~W}$ images (24). The infarct volume was calculated based on the T2W images using ImageJ software (National Institutes of Health, USA). The apparent diffusion coefficient (ADC) estimates the water diffusion in the brain (25). The relative ADC (\%) was calculated by the $\mathrm{ADC}$ values in the peri-infarct area divided by the corresponding area in the contralateral brain.

\section{Modified Garcia score test}

The sensorimotor functions in the mice were assessed using the modified Garcia test at 24 hours after dMCAO as previously described $(20,26)$. Briefly, five tests were 
conducted, including body proprioception, vibrissae touch, limb symmetry, lateral turning, and forelimb walking. For each test, the scores ranged from $0-3$.

\section{Terminal deoxynucleotidyl transferase (TdT)-mediated dUTP nick end labeling (TUNEL) staining}

TUNEL staining was conducted using the In Situ Cell Death Detection Kit according to the manufacturer's instructions (Roche, South San Francisco, CA, USA). The nuclei of cells were stained with DAPI as previously described (22).

\section{Statistical analysis}

Data are presented as mean \pm standard deviation $(\mathrm{SD})$. All statistical analyses were conducted using GraphPad Prism 8.0 software (GraphPad Software Inc., La Jolla, CA, USA). Statistical differences were analyzed using one way analysis of variance (ANOVA) or unpaired $t$-tests. A $\mathrm{P}$ value $<0.05$ was considered statistically significant.

\section{Results}

IKK $\alpha$ played a critical role in regulating autophagy under OGD/R treatment

The expression of IKK $\alpha$ and the presence of autophagy after OGD/R were investigated in vitro. The protein expression of IKK $\alpha$ was significantly increased after OGD/R (Figure $1 A, 1 B$ ). However, the expression of IKK $\beta$ did not change under OGD/R treatment (Figure $1 A, 1 C$ ). Meanwhile, the ratio of the specific autophagic key protein LC3B II/I was significantly increased in OGD/R treated N2A cells (Figure 1A,1D). Autophagic activity assessed using the commercial Cyto-ID Autophagy Detection Kit showed significant accumulation of green autophagic fluorescence signals in the perinuclear region in $\mathrm{OGD} / \mathrm{R}$ treated cells but not in control cells (Figure 1E), suggesting that autophagic activity increased after OGD/R treatment.

To explore whether IKK $\alpha$ was involved in OGD/ R-induced autophagy in vitro, IKK $\alpha$ siRNA or control siRNA was transfected into N2A cells before OGD/ $\mathrm{R}$ treatment. The LC3 II/I ratio was significantly decreased in OGD/R cells transfection with IKK $\alpha$ siRNA compared to the cells transfected with the control siRNA (Figure 1A,1D). Moreover, Cyto-ID autophagic fluorescence signals were also impaired after knockdown of IKK $\alpha$ expression (Figure 1E). Taken together, these data suggested that IKK $\alpha$ was essential for OGD/R-induced autophagy in vitro.

\section{Inbibition of IKK $\alpha$ reduced infarct volume after dMCAO}

To further clarify the effects of IKK $\alpha$ on acute stroke in vivo, IKK $\alpha$ siRNA or control siRNA were administered into the right ventricle of the mice (Figure $2 A, 2 B$ ). TTC staining at 24 hours after $\mathrm{dMCAO}$ (Figure 2C,2D) showed that the infarct volume in the IKK $\alpha$ siRNA group (infarct volume: $16.00 \pm 3.09 \mathrm{~mm}^{3}$ ) was significantly decreased compared with the control siRNA group (infarct volume: $\left.24.33 \pm 5.34 \mathrm{~mm}^{3} ; \mathrm{P}=0.0133\right)$. To further confirm the protective role of IKK $\alpha$ inhibition after $\mathrm{dMCAO}, \mathrm{T} 2 \mathrm{~W}$ MRI were performed to assess the infarct volume at 24 hours after dMCAO (Figure 2E). As shown in Figure 2F, the infarct size was significantly lower in IKK $\alpha$ siRNAtreated mice compared to control siRNA-treated ischemic mice. Moreover, increased relative ADC values in the periinfarct area of ipsilateral cortex were detected in the IKK $\alpha$ siRNA-treated group $(82.15 \% \pm 1.93 \%)$ compared to the control siRNA-treated group $(74.03 \% \pm 3.24 \% ; \mathrm{P}=0.0013$; Figure $2 G)$. rCBF was monitored before and after $\mathrm{dMCAO}$ (Figure $2 \mathrm{H}$ ). There was no significant difference in $\mathrm{rCBF}$ reduction at $24 \mathrm{~h}$ after ischemia between IKK $\alpha$ siRNAand Con siRNA-treated groups (Figure 2I). These results suggested that inhibition of IKK $\alpha$ exerted a protective effect on cerebral brain damage after $\mathrm{dMCAO}$.

\section{Inbibition of IKKa improved neurological functions after dMCAO}

To investigate the effects of IKK $\alpha$ inhibition on neurological functions, sensorimotor deficits were assessed using the modified Garcia test scores at 24 hours after dMCAO. As shown in Figure $3 A$, vibrissae touch was significantly improved in IKK $\alpha$ siRNA-treated mice compared to control siRNA-treated mice. The IKK $\alpha$ siRNA treated group showed an improved trend with four other sensorimotor functions, including body proprioception, limb symmetry, lateral turning, and forelimb walking (Figure 3B-3E). All five assessments resulted in an increase in the total neurological score in IKK $\alpha$ siRNA-mice compared to control siRNA mice (Figure $3 F$ ). These data revealed that inhibition of IKK $\alpha$ improved some aspects of neurological outcomes at 24 hours after $\mathrm{d} M C A O$. 
A

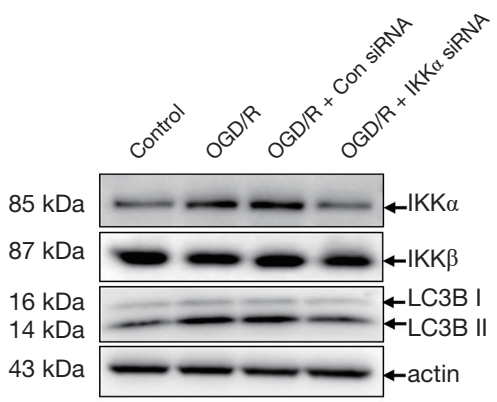

C

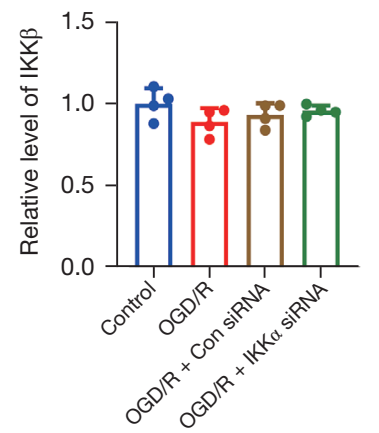

E

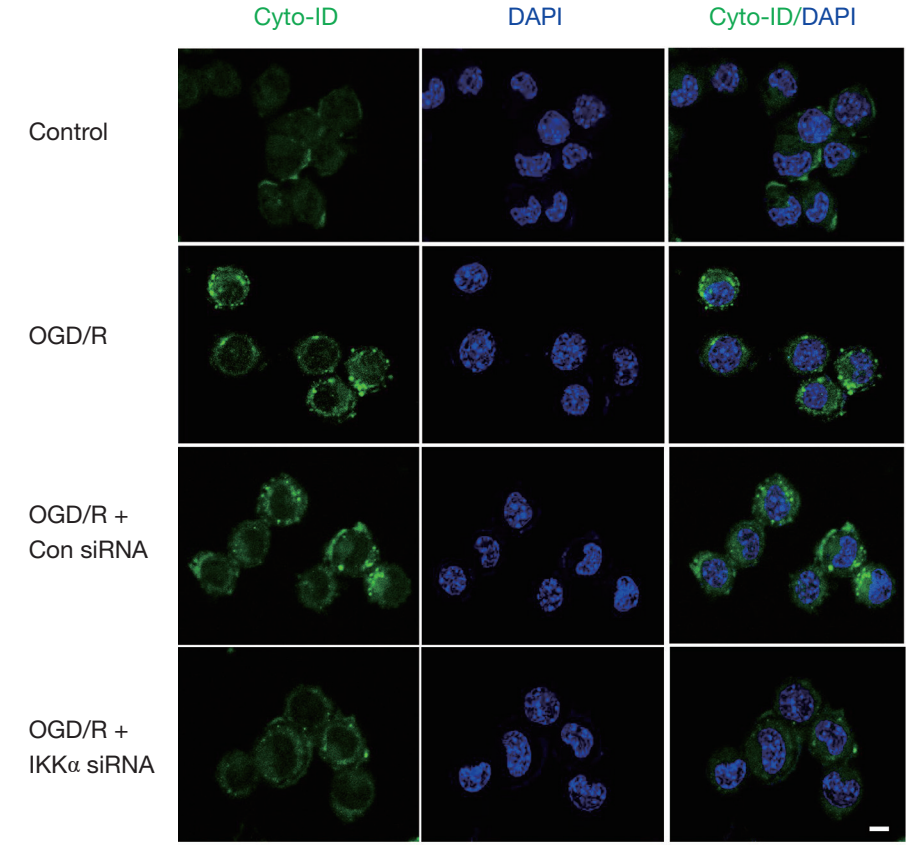

B

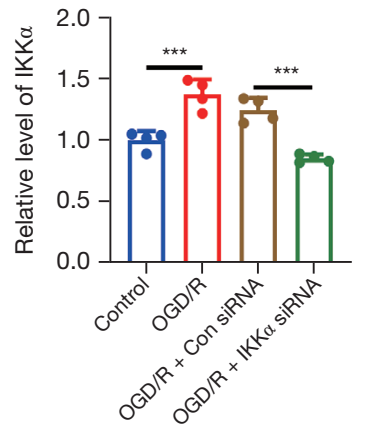

D

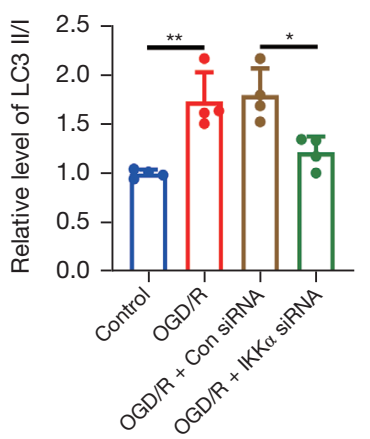

Cyto-ID/DAPI

Figure 1 The effect of IKK $\alpha$ on OGD-induced autophagy in N2A cells. (A) N2A cells were transfected with IKK $\alpha$ siRNA or control siRNA, followed by 1 hour OGD and 24 hours reoxygenation. N2A cells were treated as follows: control, OGD/R, OGD/R + control (Con) siRNA, and OGD/R + IKK $\alpha$ siRNA. The expression of IKK $\alpha$, IKK $\beta$, and LC3B II/I were examined at 24 hours. Quantitative analysis of IKK $\alpha$ (B), IKK $\beta$ (C), and LC3B II/I (D) expression. (E) Cyto-ID autophagy detection was performed in N2A. N2A cells were incubated with Cyto-ID green detection reagent (green) and DAPI (blue) nuclear stain for 30 minutes at $37^{\circ} \mathrm{C}$. Cells were then observed by confocal microscopy. Western blot assay was repeated for four times. Scale bar $=10 \mu \mathrm{m} .{ }^{*}, \mathrm{P}<0.05 ;{ }^{* *}, \mathrm{P}<0.01$; ***, $\mathrm{P}<0.001$. IKK, IкB kinase; OGD, oxygen glucose deprivation; R, reperfusion; N2A, neuro-2A; LC3B, microtubule-associated protein 1 light chain 3ß; DAPI: 4',6-diamidino2-phenylindole. 
A

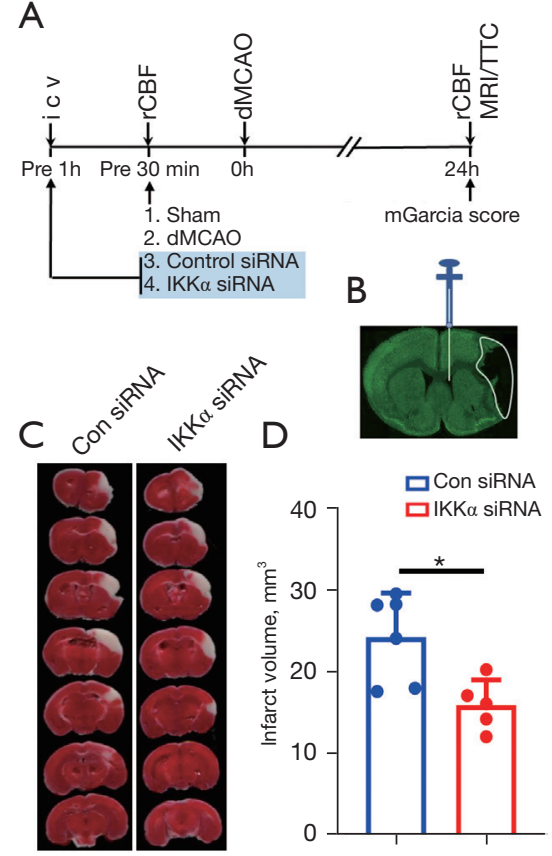

E
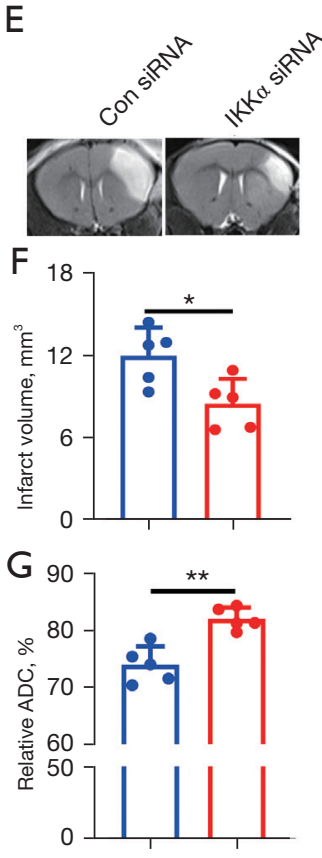

$\mathrm{H}$

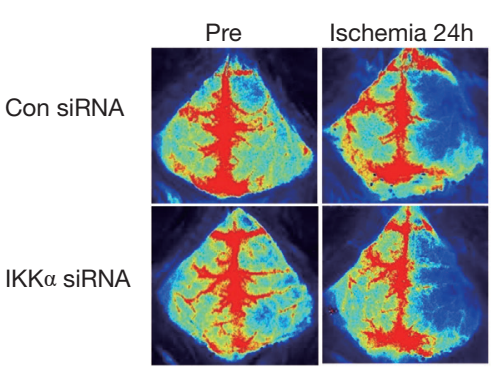

I

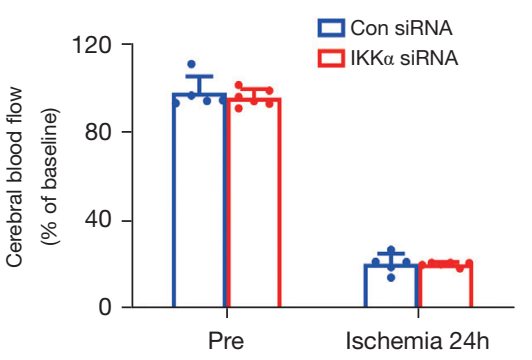

Figure 2 The effects of IKK $\alpha$ siRNA on cerebral infarct after dMCAO. (A) Timeline for the in vivo study. Permanent ischemia was induced in mice with $\mathrm{dMCAO}$. IKK $\alpha$ siRNA or control siRNA was intracerebroventricularly injected 1 hour before dMCAO onset. rCBF was assessed before and after dMCAO. TTC staining/MRI was performed to determine the infarct volume. Modified Garcia scores were conducted to measure post-ischemia neurological function at 24 hours after dMCAO. (B) The schematic map showing localization of i.c.v injection in mice. (C) Representative TTC staining at 24 hours after dMCAO. (D) The infarct volume in control siRNA and IKK $\alpha$ siRNAtreated dMCAO mice according to the TTC staining (5 to 6 mice per group). (E) Representative T2W-MRI at 24 hours after dMCAO. (F) The infarct volume was determined by T2W-MRI at 24 hours after dMCAO ( $\mathrm{n}=5$ mice per group). (G) Relative ADC (\%) was measured in control siRNA and IKK $\alpha$ siRNA-treated dMCAO mice ( $\mathrm{n}=5$ mice per group). (H) Representative images of rCBF in IKK $\alpha$ siRNA and control siRNA-treated mice. (I) Quantification of rCBF. Data are presented as a percentage of baseline (pre-dMCAO). *, $\mathrm{P}<0.05 ;$ **, $\mathrm{P}<0.01$. IKK, IкB kinase; dMCAO, distal middle cerebral artery occlusion; rCBF, regional cerebral blood flow; TTC, 2,3,5-triphenyltetrazolium chloride; MRI, magnetic resonance imaging; i.c.v, intracerebroventricular; T2W, T2 weighted; ADC, apparent diffusion coefficient.

\section{Inbibition of IKK $\alpha$ decreased cell death after dMCAO}

The protective role of IKK $\alpha$ inhibition after dMCAO was further assessed using the TUNEL assay. The percentage of TUNEL positive cells in the dMCAO group $(15.24 \% \pm 3.65 \%)$ and the control siRNA group $(17.66 \% \pm 5.02 \%)$ were significantly increased compared with the sham group $(2.11 \% \pm 2.25 \% ; \mathrm{P}<0.001$; Figure $4 A, 4 B)$. Mice treated with IKKa siRNA $(7.84 \% \pm 5.16 \%)$ showed significantly decreased TUNEL positive cells compared to the control siRNA group $(17.66 \% \pm 5.02 \%$; $\mathrm{P}<0.001$; Figure $4 A, 4 B)$. These results suggested that inhibition of IKK $\alpha$ reduced cell death after dMCAO.

\section{Inbibition of IKKa reduced autophagy activation after dMCAO}

To verify the relationship between IKK $\alpha$ and autophagy in vivo, IKKa expression levels and autophagy activation were examined using tissue from the cerebral cortex of mice after dMCAO. As shown in Figure 5A,5B, the levels of IKK $\alpha$ were significantly increased in $\mathrm{dMCAO}$ mice and control siRNA treated mice. The ratio of LC3B-II/I also upregulated after dMCAO. The IKK $\alpha$ siRNA was injected into the ipsilateral ventricle of the mice at 1 hour before the onset of ischemia and the ischemia-induced IKK $\alpha$ expression was effectively blocked after dMCAO. 
A

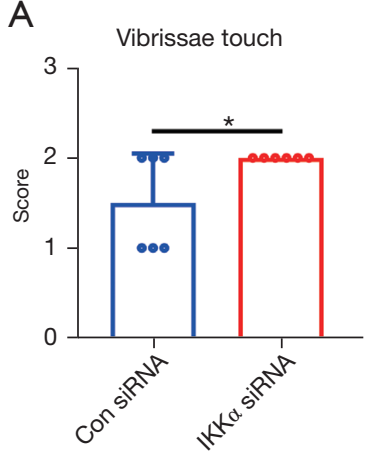

D Lataral turning

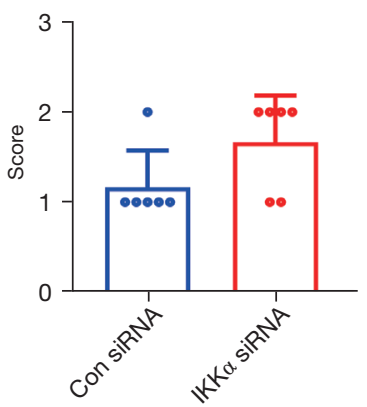

B

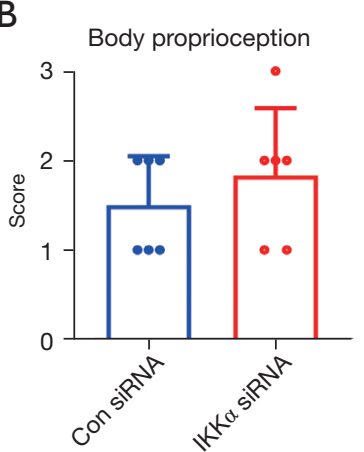

E

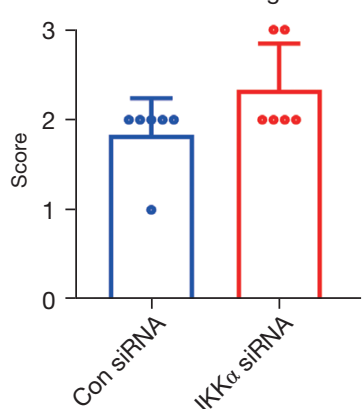

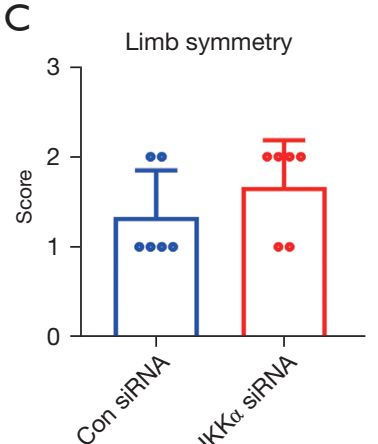

F

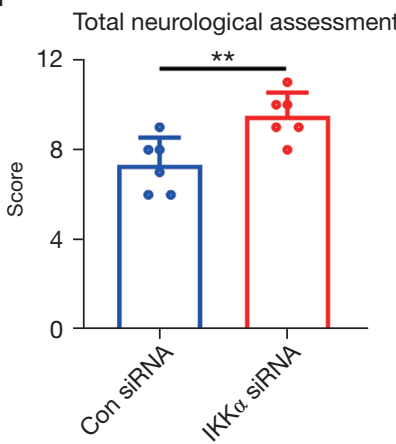

Figure 3 The effects of IKK $\alpha$ siRNA on sensorimotor functions after dMCAO. (A) Vibrissae touch. (B) Body proprioception. (C) Limb

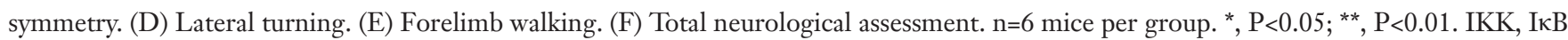
kinase; dMCAO, distal middle cerebral artery occlusion.

Under the same conditions, the conversion of LC3 I/II was significantly reduced compared with that in the control siRNA group (Figure 5A,5C). The expression of IKK $\beta$ was not altered after $\mathrm{dMCAO}$ nor after IKK $\alpha$ siRNA treatment (Figure 5A,5D).

To further determine whether IKK $\alpha$ regulates the level of autophagy after cerebral ischemia, immunostaining of LC3 was assessed. Positive fluorescence (autophagosome) was induced in $\mathrm{dMCAO}$ and control siRNA mice. In contrast, LC3 positive signals were hardly seen in the IKK $\alpha$ siRNA group (Figure 5E). These results suggested that IKK $\alpha$ was involved in ischemia-induced autophagy after cerebral ischemia.

\section{Discussion}

The role of IKK $\alpha$, one of the catalytic kinases of the I $\mathrm{B}$ kinase complex, in cerebral ischemia is not fully understood. To the best of our knowledge, this is the first study to report ischemia-induced IKK $\alpha$ upregulation both in vitro, in N2A cells during 1 hour OGD/24 hours reoxygenationinduced ischemic injury, and in vivo, in the peri-infarct area of mice at 24 hours after permanent cerebral ischemia (Figures 1,5). Under the same conditions, the expression of the other catalytic kinase of IKK (IKK $\beta$ ) did not change after ischemia. Furthermore, inhibition of IKK $\alpha$ provided neuroprotection against cerebral ischemia through decreasing LC3-mediated autophagy both in vitro and in vivo (Figures $2-5$ ).

There is accumulating evidence that IKK $\alpha$ has crucial $\mathrm{NF}-\kappa \mathrm{B}-$ dependent and NF- $\kappa \mathrm{B}$-independent functions under various physiological and pathological conditions, such as inflammation, cellular stress, and aging $(4,27,28)$. Our previous data showed that IKK $\alpha$ regulated vascular endothelial growth factor (VEGF) expression through a c-Fos-dependent, but not IKK $\beta / \mathrm{NF}-\mathrm{kB}$-independent manner in UVB-induced photodamage (7). In addition, IKK $\alpha$ has been reported to independently inhibit the transcription of maspin, a metastasis suppressor, thereby mediating tumor metastasis. Moreover, IKK $\alpha$ knockdown decreased $\beta$-catenin expression without altering NF- $\kappa \mathrm{B}$ activity and inhibited cell growth in multiple myeloma cells (4). These results revealed that IKK $\alpha$ may trigger a battery of $\mathrm{NF}-\kappa \mathrm{B}$-independent downstream substrates. The role of 


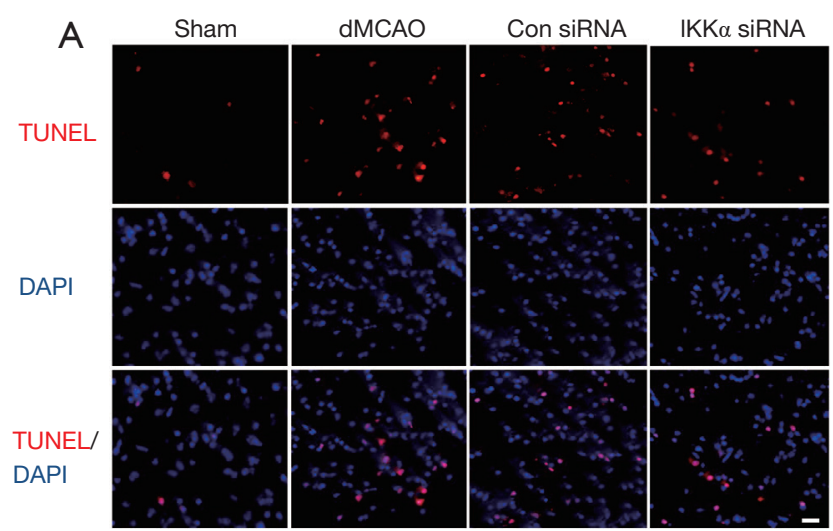

B

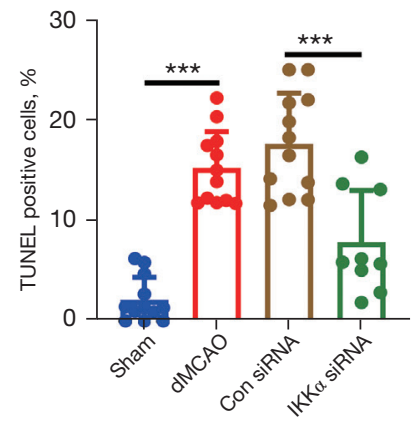

Figure 4 The effects of IKK $\alpha$ siRNA on cell death after dMCAO. (A) Representative TUNEL positive cells (red fluorescence) in brain slices from 4 groups of mice at 24 hours after dMCAO. Brain slices were incubated with TUNEL reagent (red) for 60 min at $37{ }^{\circ} \mathrm{C}$, then stained with DAPI (blue) for $30 \mathrm{~min}$. Images were performed on a fluorescence microscope. (B) Statistical analysis of the ratio of TUNEL positive cells in the 4 groups of mice. Scale bar $=20 \mu \mathrm{m} . \mathrm{n}=3$ to 4 mice per group. ${ }^{* * *}, \mathrm{P}<0.001$. IKK, IкB kinase; dMCAO, distal middle cerebral artery occlusion; DAPI, 4',6-diamidino-2-phenylindole; TUNEL, (TdT)-mediated dUTP nick end labeling.

IKK $\alpha$ in cerebral ischemia is not fully understood. After transient focal cerebral ischemia, IKK $\alpha$ activation resulted in phosphorylation of histone $\mathrm{H} 3$ in response to oxidative stress (9). IKK $\alpha$ is degraded via selective autophagy in primary microglia under OGD/R conditions (10). To improve the quality of preclinical studies, the Stroke Therapy Academic Industry Roundtable (STAIR) recommends that both transient and permanent occlusion models should be performed in the preclinical studies, especially permanent occlusion models should be adopted first (29). In the current study, IKK $\alpha$, but not IKK $\beta$, was significantly induced in N2A cells after OGD/R treatment and mice suffered permanent cerebral ischemia (Figures 1,5). Application of IKK $\alpha$ siRNA exerted a protective role against brain damage through reducing cerebral infarct volume, improving sensorimotor functions, and decreasing cell death (Figures 2-4).

The ADC measured by MRI reflects the degree of diffusion of water molecules (24). In pathological conditions, a decrease in ADC values is considered to be associated with the reduction of extracellular space caused by cellular swelling (30). As indicated in Figure 2G, knockdown of IKK $\alpha$ expression significantly increased the relative $\mathrm{ADC}$ in the peri-infarct area at 24 hours after dMCAO. This result demonstrated that IKK $\alpha$ may contribute to cell death through altered water mobility and related cytotoxic edema after acute cerebral ischemia. However, the relationship between IKK $\alpha$ and cell swelling warrants further investigation.
In response to nutrient deprivation, IKK $\alpha$ induces the expression of the essential pro-autophagic gene, Beclin-1. Moreover, loss of NF-кB does not affect starvation-induced expression of the autophagic gene (31). However, a previous study from phosphatase and tensin homolog deleted on chromosome ten (PTEN)-null prostate cancer cells showed that IKK $\alpha$ controlled mTOR activation, which in turn inhibited autophagy, with less involvement by IKK $\beta$ (4). Thus, the role of IKK $\alpha$ in regulating autophagy may vary in different settings.

The potential mechanisms by which IKK $\alpha$ mediates ischemia-induced LC3B expression is unclear at present. It is possible that IKK $\alpha$ directly binds with LC3B through the classical LC3-interacting region (LIR) motif (W/F/ $\mathrm{Y}-\mathrm{X}-\mathrm{X}-\mathrm{V} / \mathrm{L} / \mathrm{I}$ ) (32). AMP-activated protein kinase (AMPK) is a vital sensor of ATP/ADP energy balance in cells and triggers autophagy when activated (33). Inhibiting the expression of IKK decreases the activation of AMPK and the conversion of LC3-I to LC3-II in intestinal epithelial cells. We observed AMPK phosphorylation in the periinfarct area of mice with acute cerebral ischemia (data not shown). It is therefore possible that the association between IKK $\alpha$ and LC3B is mediated by AMPK and warrants further investigation.

\section{Conclusions}

In conclusion, this study clearly elucidated a novel role of IKK $\alpha$ in regulating the activation of autophagy after 


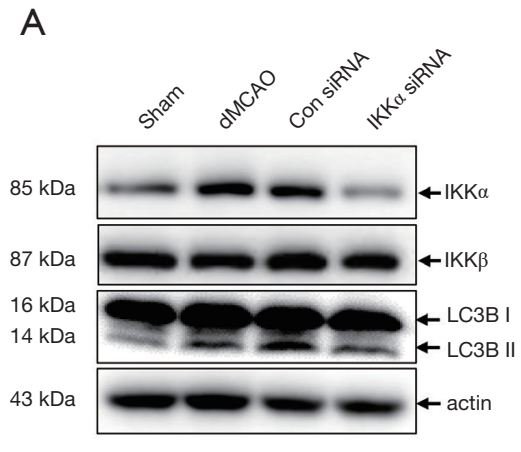

B

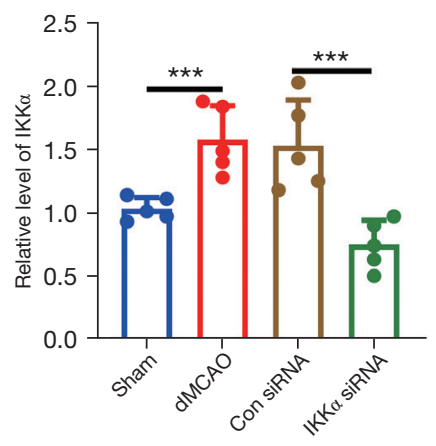

C

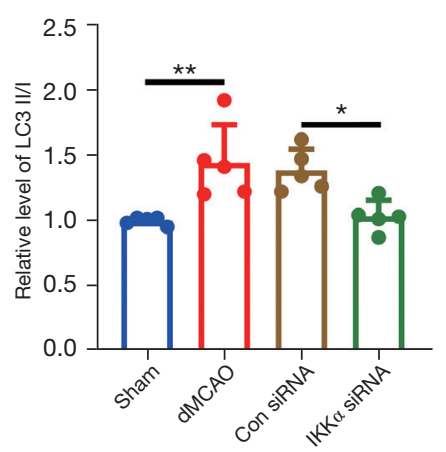

E

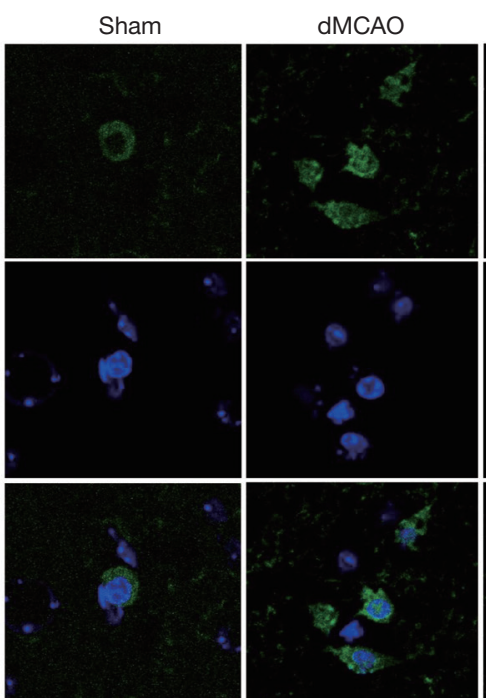

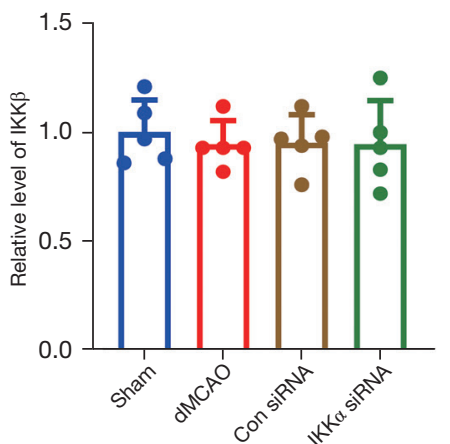

Con siRNA

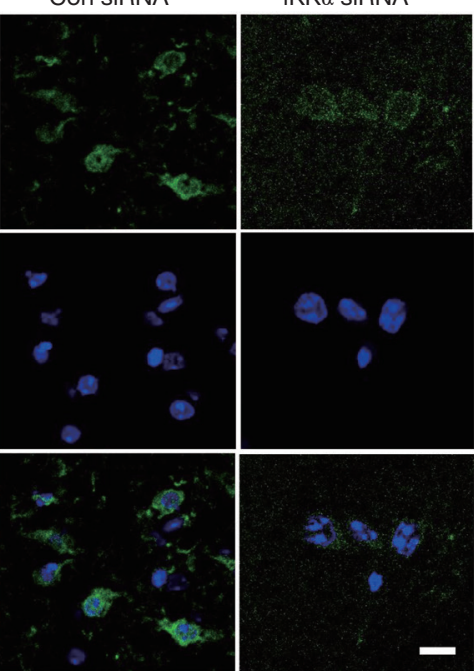

Figure 5 The effects of IKK $\alpha$ siRNA on autophagy activation after dMCAO. (A) Western blot showing the representative proteins bands of IKK $\alpha$, IKK $\beta$, and LC3B at 24 hours after dMCAO. (B-D) Statistical quantification of IKK $\alpha$, LC3B II/I, and IKK $\beta$ detected by Western blot ( $\mathrm{n}=5$ per group). * $\mathrm{P}<0.05 ;{ }^{* *}, \mathrm{P}<0.01$; ${ }^{* * *}, \mathrm{P}<0.001$. (E) Representative images showing the autophagosomes (LC3 positive cells, green) at 24 hours after dMCAO. Scale bar $=10 \mu \mathrm{m}$. Brain sections were co-stained with LC3 (green) and DAPI (blue) for $1 \mathrm{~h}$ at room temperature. DAPI is used as a nuclear marker. Images were then performed on a confocal microscope. IKK, IкB kinase; dMCAO, distal middle cerebral artery occlusion; LC3B, microtubule-associated protein 1 light chain 3ß; DAPI, 4',6-diamidino-2-phenylindole. 
acute cerebral ischemia. These finding may assist in the development of novel therapeutic strategies for alleviating brain injury by targeting IKK $\alpha$.

\section{Acknowledgments}

Funding: This work was supported by the National Natural Science Foundation of China (Nos. 81701138, 81871021, 82171268); the Beijing Hospitals Authority Youth Programme (No. QML20180505); the Beijing Excellent Talents (No. 2017000021469G249); and the National Science and Technology Major Project (No. 2017ZX09304018).

\section{Footnote}

Reporting Checklist: The authors have completed the ARRIVE reporting checklist. Available at https://atm. amegroups.com/article/view/10.21037/atm-22-517/rc

Data Sharing Statement: Available at https://atm.amegroups. com/article/view/10.21037/atm-22-517/dss

Conflicts of Interest: All authors have completed the ICMJE uniform disclosure form (available at https://atm. amegroups.com/article/view/10.21037/atm-22-517/coif). The authors have no conflicts of interest to declare.

Ethical Statement: The authors are accountable for all aspects of the work in ensuring that questions related to the accuracy or integrity of any part of the work are appropriately investigated and resolved. All experimental procedures were performed under a project license (No. 201902039) by the Animal Welfare Ethics Committee of Beijing Neurosurgical Institute and were performed in accordance with the guidelines of the National Institutes of Health on the care and use of laboratory animals.

Open Access Statement: This is an Open Access article distributed in accordance with the Creative Commons Attribution-NonCommercial-NoDerivs 4.0 International License (CC BY-NC-ND 4.0), which permits the noncommercial replication and distribution of the article with the strict proviso that no changes or edits are made and the original work is properly cited (including links to both the formal publication through the relevant DOI and the license). See: https://creativecommons.org/licenses/by-nc-nd/4.0/.

\section{References}

1. Wang YJ, Li ZX, Gu HQ, et al. China Stroke Statistics 2019: A Report From the National Center for Healthcare Quality Management in Neurological Diseases, China National Clinical Research Center for Neurological Diseases, the Chinese Stroke Association, National Center for Chronic and Non-communicable Disease Control and Prevention, Chinese Center for Disease Control and Prevention and Institute for Global Neuroscience and Stroke Collaborations. Stroke Vasc Neurol 2020;5:211-39.

2. Thiebaut AM, Gauberti M, Ali C, et al. The role of plasminogen activators in stroke treatment: fibrinolysis and beyond. Lancet Neurol 2018;17:1121-32.

3. Hacke W, Kaste M, Bluhmki E, et al. Thrombolysis with alteplase 3 to 4.5 hours after acute ischemic stroke. $\mathrm{N}$ Engl J Med 2008;359:1317-29.

4. Hinz $M$, Scheidereit C. The IкB kinase complex in NF- $\kappa B$ regulation and beyond. EMBO Rep 2014;15:46-61.

5. Chariot A. The NF-kappaB-independent functions of IKK subunits in immunity and cancer. Trends Cell Biol 2009;19:404-13.

6. Huang WC, Hung MC. Beyond NF- $\kappa B$ activation: nuclear functions of IкB kinase $\alpha$. J Biomed Sci 2013;20:3.

7. Dong W, Li Y, Gao M, et al. IKK $\alpha$ contributes to UVB-induced VEGF expression by regulating AP-1 transactivation. Nucleic Acids Res 2012;40:2940-55.

8. Herrmann O, Baumann B, de Lorenzi R, et al. IKK mediates ischemia-induced neuronal death. Nat Med 2005;11:1322-9.

9. Song YS, Kim MS, Kim HA, et al. Oxidative stress increases phosphorylation of IkappaB kinase-alpha by enhancing NF-kappaB-inducing kinase after transient focal cerebral ischemia. J Cereb Blood Flow Metab 2010;30:1265-74.

10. Li X, Xia Q, Mao M, et al. Annexin-A1 SUMOylation regulates microglial polarization after cerebral ischemia by modulating IKK $\alpha$ stability via selective autophagy. Sci Adv 2021;7:eabc5539.

11. Abounit K, Scarabelli TM, McCauley RB. Autophagy in mammalian cells. World J Biol Chem 2012;3:1-6.

12. Yao RQ, Ren C, Xia ZF, et al. Organelle-specific autophagy in inflammatory diseases: a potential therapeutic target underlying the quality control of multiple organelles. Autophagy 2021;17:385-401.

13. Li W, He P, Huang Y, et al. Selective autophagy of intracellular organelles: recent research advances. 
Theranostics 2021;11:222-56.

14. Damme M, Suntio T, Saftig P, et al. Autophagy in neuronal cells: general principles and physiological and pathological functions. Acta Neuropathol 2015;129:337-62.

15. Loos B, du Toit A, Hofmeyr JH. Defining and measuring autophagosome flux - concept and reality. Autophagy 2014;10:2087-96.

16. Wang $\mathrm{P}$, Shao BZ, Deng Z, et al. Autophagy in ischemic stroke. Prog Neurobiol 2018;163-164:98-117.

17. Papadakis M, Hadley G, Xilouri M, et al. Tsc1 (hamartin) confers neuroprotection against ischemia by inducing autophagy. Nat Med 2013;19:351-7.

18. Wang P, Guan YF, Du H, et al. Induction of autophagy contributes to the neuroprotection of nicotinamide phosphoribosyltransferase in cerebral ischemia. Autophagy 2012;8:77-87.

19. Jiang WW, Huang BS, Han Y, et al. Sodium hydrosulfide attenuates cerebral ischemia/reperfusion injury by suppressing overactivated autophagy in rats. FEBS Open Bio 2017;7:1686-95.

20. Dong $W$, Zhao $S$, Wen $S$, et al. A preclinical randomized controlled study of ischemia treated with Ginkgo biloba extracts: Are complex components beneficial for treating acute stroke? Curr Res Transl Med 2020;68:197-203.

21. Liu X, Liu J, Zhao S, et al. Interleukin-4 Is Essential for Microglia/Macrophage M2 Polarization and Long-Term Recovery After Cerebral Ischemia. Stroke 2016;47:498-504.

22. Liu X, Wen S, Zhao S, et al. Mild Therapeutic Hypothermia Protects the Brain from Ischemia/ Reperfusion Injury through Upregulation of iASPP. Aging Dis 2018;9:401-11.

23. Dong W, Qi Z, Liang J, et al. Reduction of zinc accumulation in mitochondria contributes to decreased cerebral ischemic injury by normobaric hyperoxia

Cite this article as: Dong W, Chen Q, Zhao S, Wen S, Chen W, Ye W, Gong T, Jiang M, Liu X. IKK $\alpha$ contributes to ischemiainduced autophagy after acute cerebral ischemic injury. Ann Transl Med 2022;10(4):160. doi: 10.21037/atm-22-517 treatment in an experimental stroke model. Exp Neurol 2015;272:181-9.

24. Xu R, Bai Y, Min S, et al. In vivo Monitoring and Assessment of Exogenous Mesenchymal Stem CellDerived Exosomes in Mice with Ischemic Stroke by Molecular Imaging. Int J Nanomedicine 2020;15:9011-23.

25. Ohene $Y$, Harrison IF, Nahavandi P, et al. Non-invasive MRI of brain clearance pathways using multiple echo time arterial spin labelling: an aquaporin-4 study. Neuroimage 2019;188:515-23.

26. Liu Z, Ran Y, Huang S, et al. Curcumin Protects against Ischemic Stroke by Titrating Microglia/Macrophage Polarization. Front Aging Neurosci 2017;9:233.

27. Tilstra JS, Clauson CL, Niedernhofer LJ, et al. NF- $\kappa$ B in Aging and Disease. Aging Dis 2011;2:449-65.

28. Antonia RJ, Hagan RS, Baldwin AS. Expanding the View of IKK: New Substrates and New Biology. Trends Cell Biol 2021;31:166-78.

29. Fisher M, Feuerstein G, Howells DW, et al. Update of the stroke therapy academic industry roundtable preclinical recommendations. Stroke 2009;40:2244-50.

30. Badaut J, Ashwal S, Adami A, et al. Brain water mobility decreases after astrocytic aquaporin-4 inhibition using RNA interference. J Cereb Blood Flow Metab 2011;31:819-31.

31. Comb WC, Cogswell P, Sitcheran R, et al. IKKdependent, NF- $\mathrm{KB}$-independent control of autophagic gene expression. Oncogene 2011;30:1727-32.

32. Petherick KJ, Williams AC, Lane JD, et al. Autolysosomal $\beta$-catenin degradation regulates Wnt-autophagy-p62 crosstalk. EMBO J 2013;32:1903-16.

33. Li Y, Chen Y. AMPK and Autophagy. Adv Exp Med Biol 2019;1206:85-108. 